\title{
Correction to: Genetic characteristics of the Iranian honey bee, Apis mellifera meda, based on mitochondrial genes of ND4, ND4L and ND6 and their internal transcribed spacers
}

\author{
H. Veisi ${ }^{1}$ - J. Nazemi-Rafie ${ }^{1}$ - A. Azizi ${ }^{1} \cdot$ H. Rajabi-Maham ${ }^{2}$
}

Published online: 19 June 2020

(c) International Union for the Study of Social Insects (IUSSI) 2020

\section{Correction to: Insectes Sociaux}

https://doi.org/10.1007/s00040-020-00769-2

While typesetting the article Table column head has been incorrectly published.

The correct Table 1 has been copied below.

The original article can be found online at https://doi.org/10.1007/ s00040-020-00769-2.

J. Nazemi-Rafie

j.nazemi@uok.ac.ir

1 Plant Protection Department, Faculty of Agriculture,

University of Kurdistan, Sanandaj, Kurdistan, Iran

2 Department of Animal Sciences and Biotechnology, Faculty

of Life Sciences and Biotechnology, Shahid Beheshti

University G.C., Tehran, Iran 
Table 1 The evolutionary lineages of A. mellifera based on morphological and molecular data

\begin{tabular}{|c|c|c|c|}
\hline A lineage & C lineage & M lineage & O lineage \\
\hline \multicolumn{4}{|c|}{ The evolutionary lineages based on morphological data } \\
\hline A. m. jemenitica & A. m. carnica & A. m. mellifera & A. m. caucasica \\
\hline A. m. intermissa & A. m. ligustica & A. m. iberiensis & A. m. anatoliaca \\
\hline A. m. monticola & & & A. m. meda \\
\hline A. m. scutellata & & & A. m. syriaca \\
\hline A. m. adansonii & & & \\
\hline A. m. capensis & & & \\
\hline A. m. litorea & & & \\
\hline A. m. lamarckii & & & \\
\hline A lineage & $\mathrm{C}$ lineage & M linegae & Z subgroup \\
\hline \multicolumn{4}{|c|}{ The evolutionary lineages based on molecular data } \\
\hline A. m. intermissa & A. m. caucasica & A. m. mellifera & A. m. syriaca \\
\hline A. m. monticola & A. m. anatoliaca & A. m. iberiensis & A. m. jemenitica \\
\hline A. m. scutellata & A. m. meda & A. m. sinisxinyuan & A. m. lamarckii \\
\hline A. m. adansonii & A. m. carnica & & \\
\hline A. m. capensis & A. m. ligustica & & \\
\hline A. m. litorea & & & \\
\hline
\end{tabular}

The original article has been corrected. 